\title{
Bufadienolides induce apoptosis and autophagy by inhibiting the AKT signaling pathway in melanoma A-375 cells
}

\author{
HUANNAN LI*, XIAOREN CAO*, XIAOJUAN LIN, XIONGBING CHEN, \\ XIAOHONG YI, JUN XIA, JILAN CHEN and LAN YANG \\ Department of Anatomy, Histology and Embryology, Chengdu University of Traditional Chinese Medicine, \\ Chengdu, Sichuan 610075, P.R. China
}

Received September 5, 2018; Accepted April 4, 2019

DOI: $10.3892 / \mathrm{mmr} .2019 .10452$

\begin{abstract}
The purpose of the present study was to investigate the effect of bufadienolides on the A-375 melanoma cell line, and to delineate the underlying mechanism. A Cell Counting Kit-8 assay was used to determine the viability of the cells, and flow cytometry was used to evaluate apoptosis. Western blot analysis was used to evaluate the expression levels of proteins involved in the AKT pathway that are associated with apoptosis and autophagy. The results demonstrated that bufadienolides reduced the viability of A-375 cells in a dose- and a time-dependent manner. Following treatment with bufadienolides, A-375 cells exhibited clear properties that were characteristic of apoptosis and autophagy. The expression levels of the pro-apoptotic proteins Bax and p53 were upregulated, whereas those of the anti-apoptotic proteins, Bcl-2 and caspase- 3 were downregulated. In addition, the level of a protein known to be associated with autophagy, microtubule-associated proteins 1A/1B light chain 3-II, was increased, whereas that of p62 protein was reduced. Finally, the AKT signaling pathway was blocked in the bufadienolide-treated A-375 cells. In conclusion, these results revealed that bufadienolides effectively induced apoptosis and autophagy in A-375 cells via the AKT pathway, and therefore may be one of the candidate targets for the future development of targeted drugs to treat melanoma.
\end{abstract}

Correspondence to: Professor Lan Yang, Department of Anatomy, Histology and Embryology, Chengdu University of Traditional Chinese Medicine, 1166 Liutai Avenue, Wenjiang, Chengdu, Sichuan 610075, P.R. China

E-mail: lanyang72@163.com

*Contributed equally

Key words: bufadienolide, malignant melanoma, A-375 cells, apoptosis, autophagy, AKT signaling pathway

\section{Introduction}

Melanoma, also known as malignant melanoma, arises from the pigment-producing cells of the deeper layers of the skin, attributed to skin lesions (1). Melanocytes are abundant in the skin (2). Melanogenesis is complex regulated by a variety of factors that interact with each other in a hormonal, autogenous, para-endocrine or endocrine manner, through receptor-dependent and independent mechanisms of activation (3). Nearly 20,000 cases of cutaneous malignant melanoma are diagnosed in China every year (4). Increasing numbers of studies have confirmed that ultraviolet (UV) radiation is the main risk factor for the development of melanoma. Cumulative UV exposure leads to UV-induced DNA damage, oxidative stress and skin inflammation (5-8); in particular, UV leads to DNA damage and immunosuppression, both of which cause melanoma to develop and are involved in the pathogenesis of melanoma (9). At the same time, the interaction between melanoma cells and the microenvironment of melanocytes in the skin epidermis is also considered to affect melanin damage $(10,11)$. In addition, an immunosuppressive field is produced in the intermediate stage of melanogenesis, which weakens the effect of any immunotherapy for melanoma. Therefore, uncontrolled melanogenesis, together with melanin, leads to decreased efficacy of radiotherapy, chemotherapy, phototherapy and immunotherapy (12). In view of the above, traditional treatment methods include surgical treatment, radiotherapy and chemotherapy. These treatment strategies are often self-limiting, achieve far from satisfactory results, and may even lead to an increase in the burden of global public health $(13,14)$. Therefore, the urgent task is to find an effective drug, improve the level of comprehensive treatment, reduce the side effects of melanoma and reduce the mortality rate of melanoma.

$\mathrm{Chan} \mathrm{Su}$, which is obtained from the skin and parotid venom glands of the toad (15), has been traditionally used for the treatment of a variety of clinical diseases in China. Bufadienolides, including gamabufotalin, arenobufagin, telocinobufagin, bufalin and other ingredients, are the major pharmacological constituents of Chan Su, which is frequently used in the clinic for the treatment of cancer, including 
hepatoma, gallbladder carcinoma and lung cancer (16). In previously published studies, bufadienolide compounds have been demonstrated to induce arrest of the tumor cell cycle and apoptosis $(17,18)$. In addition, bufadienolides were revealed to markedly inhibit proliferation and induce autophagy in liver cancer cells (19), and alterations in the expression levels of microtubule-associated proteins $1 \mathrm{~A} / 1 \mathrm{~B}$ light chain $3-\mathrm{II}$ (LC3-II), p62, Bax, Bcl-2, cyclin D1 and caspase-3 are involved in the underlying mechanism (20).

It has been previously reported in the literature that the AKT pathway has an important role in promoting cell apoptosis and autophagy (21). Based on these previous findings, the present study aimed to explore whether bufadienolides are able to impair the viability of melanoma cells and to increase the levels of apoptosis and autophagy via inhibition of the AKT pathway.

\section{Materials and methods}

Reagents and antibody. The bufadienolides were a gift from Dr Jiang (Macau University of Science and Technology). Preparation of bufotoxin and bufogenin fractions from toad venom and toad skin (200 g; Xiangshui Bolin Pharmaceutical \& Chemical Co., Ltd.) was ground into powder and ultrasonically extracted with methanol five times, as previously described (22). The methanol solution was filtered, combined and evaporated at reduced pressure to obtain the total extract (138 g bufadienolides; purity $>97 \%$ ), which was used for this study. The chemical profile and structure of bufadienolides has been presented in a previous study (22). Bufadienolides were dissolved in anhydrous DMSO at a concentration of $5,000 \mathrm{~g} / \mathrm{l}$, for use as a stock solution. The Annexin V-FITC \& PI Apoptosis Detection kit (cat. no. BD556547) was purchased from BD Biosciences. Anti-Bax (cat. no. 2772), anti-Bcl-2 (cat. no. 15071), anti-caspase-3 (cat. no. 9662), anti-p53 (cat. no. sc-126), anti-LC3-II (cat. no. 2775), anti-p62 (cat. no. 5114), anti-AKT (cat. no. 9272), anti-mTOR (cat. no. 2972), anti-phosphorylated (p)-AKT (Ser473; cat. no. 4060), anti-p70S6K (cat. no. 9202), anti-p-p70S6K (cat. no. 9204), anti-glycogen synthase kinase (GSK)-3 $\beta$ (cat. no. 9325), anti-p-GSK-3 $\beta$ (cat. no. (Ser9)9323) and anti-cyclinD1 (cat. no. 2922) were purchased from Cell Signaling Technology, Inc. GAPDH (cat. no. AP0066) antibody was purchased from Bioworld Technology, Inc. Horseradish peroxidase (HRP)-labeled anti-mouse IgG (cat. no. TA130001) or anti-rabbit IgG (cat. no. TA130015) were obtained from OriGene Technologies, Inc.

Cell culture and treatment. Melanoma A-375 cells, obtained from the American Type Culture Collection (cat. no. CRL-1619), were incubated in 10\% FBS (Ausbian) and Gibco ${ }^{\circledR}$ DMEM (Thermo Fisher Scientific, Inc.), and subsequently maintained at $37^{\circ} \mathrm{C}$ in an atmosphere of $5 \%$ $\mathrm{CO}_{2}$. The bufadienolides were dissolved in anhydrous DMSO, and diluted with fresh medium to achieve the desired concentrations (see below).

Cell morphology. Cells were seeded at $2 \times 10^{4} / 100 \mu 1$ per well in $500 \mu 1$ DMEM in a 24 -well plate and incubated for $12 \mathrm{~h}$. Subsequently, DMEM medium alone (negative control) and media containing bufadienolides at various concentrations $(0,0.001,0.01,0.1,1 \mathrm{mg} / \mathrm{l})$ were incubated for $0,24,48,72$ or $96 \mathrm{~h}$. The cell morphology change was visualized and photographed under an inverted phase contrast microscope at x200 magnification.

Cell Counting Kit-8 (CCK-8) assay. A-375 cells were seeded in 96 -well plates at a density of $2 \times 10^{4} / 100 \mu$ l containing $0,0.001,0.01,0.1$ and $1 \mathrm{mg} / 1$ bufadienolides, and subsequently cultured for $0,24,48,72$ or $96 \mathrm{~h}$. As a negative control, cells were incubated in the absence of bufadienolides. In order to measure cell viability, $10 \mu \mathrm{lCCK}-8$ reagent (Dojindo Molecular Technologies, Inc.) was added to each well and incubated for $2 \mathrm{~h}$ at $37^{\circ} \mathrm{C}$ in a tissue culture hood. The absorbance [optical density (OD)] at $490 \mathrm{~nm}$ was measured using a microplate reader.

Annexin V/propidium iodide (PI) staining assay. An Annexin V-FITC/PI kit was used to assess cell apoptosis. After treating with bufadienolides $(0,0.001,0.01,0.1$ and $1 \mathrm{mg} / \mathrm{l})$ for 48 h, A-375 cells were collected, and the harvested cells were diluted with PBS to a concentration of $5 \times 10^{5}-1 \times 10^{6}$ cells $/ \mathrm{ml}$, centrifuged at $4^{\circ} \mathrm{C}$ and $7,000 \mathrm{x}$ g for $10 \mathrm{~min}$, and resuspended in $200 \mu \mathrm{l} 1 \mathrm{X}$ binding buffer. Subsequently, Annexin V-FITC $(10 \mu \mathrm{l})$ and PI $(5 \mu \mathrm{l})$ were added to each sample. The samples were incubated at room temperature for $15 \mathrm{~min}$, and examined immediately on a flow cytometer (BD Biosciences), according to the manufacturer's protocol. A BD FACSCanto running BD CellQuest ${ }^{\mathrm{TM}}$ software version 3.3 (BD Biosciences) was used to perform flow cytometric analysis. Early apoptosis was defined by Annexin V+/PI-staining (Q4) and late apoptosis was defined by Annexin V+/PI+ staining (Q2).

Gel electrophoresis and western blot analysis. The expression levels of Bax, Bcl-2, caspase-3, p53, LC3-II, p62, AKT, p-AKT, mTOR, p70-S6K1, p-p70-S6K1, GSK-3 $\beta$, p-GSK-3 $\beta$ and cyclin D1 in cells were determined using western blot analysis. A-375 cells were placed in 6-well cell culture plates at a concentration of $2 \times 10^{5}$ cells/well, and incubated at $37^{\circ} \mathrm{C}$ for $24 \mathrm{~h}$. The cells were subsequently treated with bufadienolides at the aforementioned concentrations for 48 h. Subsequently, A-375 cells were lysed using RIPA lysis buffer (Beijing Solarbio Science \& Technology Co., Ltd.) on ice for $30 \mathrm{~min}$. The protein concentration was determined using a bicinchoninic acid protein assay kit (Beyotime Institute of Biotechnology). An equal amount of total protein $(20 \mu \mathrm{g})$ was loaded in each lane. Proteins were separated by $10 \%$ SDS-PAGE, the proteins were transferred onto PVDF membranes (EMD Millipore). The membranes were blocked with $5 \%$ skimmed milk powder solution at room temperature for $1 \mathrm{~h}$ in order to reduce the non-specific background. The blotted membranes were incubated with anti-Bax, anti-GAPDH, anti-Bcl-2, anti-caspase-3, anti-p53, anti-LC3-II, anti-p62, anti-AKT, anti-mTOR, anti-p-AKT, anti-p70S6K, anti-p-p70S6K, anti-GSK-3 $\beta$, anti-p-GSK-3 $\beta$ and anti-cyclin D1 (all 1:1,000) at $4^{\circ} \mathrm{C}$ overnight in the fridge. Subsequently, the membrane was washed three times with $1 \mathrm{X}$ TBS with Tween-20 (TBST), and HRP-labeled anti-mouse IgG or anti-rabbit IgG were used as secondary antibodies $(1: 10,000)$ for $2 \mathrm{~h}$ at room temperature. Membranes were 
subsequently washed again with $1 \mathrm{X}$ TBST buffer, and the protein signals were detected with an ECL kit (EMD Millipore). Protein expression levels were quantified by using a Bio-Rad Image station and its built-in software (version 6.0; Bio-Rad Laboratories, Inc.), and are shown as the densitometric ratio of the target protein to the input control, GAPDH.

Statistical analysis. Each experiment was repeated three times. All the presented data are shown as the mean \pm standard deviation. Statistical analyses were performed using one-way ANOVA followed by the Bonferroni post-hoc test in GraphPad Prism software (version 6.0; GraphPad Software, Inc.). $\mathrm{P}<0.05$ was considered to indicate a statistically significant difference.

\section{Results}

Bufadienolides inhibit the viability of the A-375 melanoma cells. The A-375 cells were treated with the various concentrations of bufadienolides for $24,48,72$ and $96 \mathrm{~h}$. The $\mathrm{IC}_{50}$ values at 24, 48, 72 and $96 \mathrm{~h}$ were $2.56,1.27,0.24$ and $0.04 \mathrm{mg} / \mathrm{l}$ (Table I). The cell morphology following bufadienolide treatment was observed. From the inverted phase contrast microscopy (Fig. 1A), it was found that following bufadienolide treatment, the A-375 cells appeared abnormal with a rounded shape; some cells had shrunk and had ruptured membranes, compared with those in the control group. Additionally, the proportion of normal and living cells decreased with an increasing bufadienolide concentration. The results of the CCK- 8 assay revealed that at $24,48,72$ and $96 \mathrm{~h}$, bufadienolide treatment led to a decrease in the viability of the A-375 cells in a time-dependent manner compared with the negative control (Fig. 1B). Furthermore, when the A-375 cells were treated with bufadienolides at different doses, their viability was inhibited in a dose-dependent manner (Fig. 1C). Therefore, these findings confirmed that bufadienolides inhibited the viability of A-375 cells in a dose- and time-dependent manner.

Bufadienolides regulate apoptosis-associated protein levels and promote apoptosis in A-375 cells. Subsequently, the present study assessed whether the bufadienolides were able to induce apoptosis in A-375 cells. It was identified that the increases in the percentages of cells in the early and late apoptotic phases were observed with an increasing concentration of bufadienolides (Fig. 2A). The cell apoptosis rates in the bufadienolide-treated cells $(0,0.001,0.01,0.1$ and $1 \mathrm{mg} / \mathrm{l})$ were $1.40 \pm 0.26,7.30 \pm 1.67,17.20 \pm 0.38,23.40 \pm 1.80$ and $28.16 \pm 0.90 \%$ (Fig. 2B). In addition, based on the results of the western blots shown, it was observed that the expression levels of Bcl-2 and caspase- 3 were reduced in a dose-dependent manner. However, conversely, the expression levels of Bax and p53 were increased in A-375 cells following treatment with bufadienolides (Fig. 2C). It was also observed that higher concentrations of the bufadienolides exerted more marked effects; in particular, the protein expression levels of Bax and p53 were appreciably enhanced (Fig. 2D). These observations suggested that bufadienolides are able to induce and promote apoptosis in A-375 cells.
Table I. $\mathrm{IC}_{50}$ values of bufadienolides in A375 cells at different time points.

\begin{tabular}{lll}
\hline Time & IC $_{50}$ value & Mean \pm SD \\
\hline $24 \mathrm{~h}$ & $2.56 \mathrm{mg} / \mathrm{l}$ & $2.56 \pm 0.70$ \\
$48 \mathrm{~h}$ & $1.27 \mathrm{mg} / \mathrm{l}$ & $1.27 \pm 0.13$ \\
$72 \mathrm{~h}$ & $0.24 \mathrm{mg} / \mathrm{l}$ & $0.24 \pm 0.54$ \\
$96 \mathrm{~h}$ & $0.04 \mathrm{mg} / \mathrm{l}$ & $0.04 \pm 0.01$ \\
\hline
\end{tabular}

Mean $\pm \mathrm{SD}$, mean \pm standard deviation

Bufadienolides regulate autophagy-associated proteins in A-375 cells. To investigate whether the inhibition of viability of the A-375 cells by bufadienolides was mediated via the potentiation of autophagy, A-375 cells were treated with bufadienolides at the specified concentrations $(0.001,0.01,0.1$ and $1 \mathrm{mg} / \mathrm{l})$ for $24 \mathrm{~h}$, and the level of autophagy was determined by western blot analysis. These experiments revealed that treatment with bufadienolides affected the protein expression levels of LC3-II and p62 in A-375 cells. It was noteworthy that the level of LC3-II was significantly increased, whereas that of p62 was reduced (Fig. 3). Furthermore, treatment with the higher concentrations of bufadienolides led to more pronounced changes in the expression levels of the autophagy-associated proteins LC3-II and p62 in A-375 cells.

Effects of bufadienolides on the AKT signaling pathway. To address whether the AKT signaling pathway is involved in mediating the effects of bufadienolide treatment on A-375 cells, the AKT pathway and its downstream proteins were investigated by western blot analysis. No evident changes in AKT expression were identified, whereas the level of p-AKT was decreased upon treatment with different concentrations of bufadienolides in the A-375 cells. There were also reductions observed in the expression levels of p-p70S6K and p-GSK-3 $\beta$, while levels of total p70S6K and GSK-3 $\beta$ were not significantly changed after exposure to different concentrations of bufadienolides. In addition, following treatment with higher concentrations of bufadienolides, the total mTOR and cyclin D1 levels were markedly decreased (Fig. 4). Each bar in the histograms represent the average of three replicates. These findings confirmed that the effect of bufadienolides on A-375 cells may be mediated via the regulation of different proteins downstream of AKT.

\section{Discussion}

Melanoma, a malignant tumor of melanocytes, is the underlying cause of $80 \%$ of mortalities associated with skin cancer (23). In this study, it was found that bufadienolides induced apoptosis and autophagy in A-375 cells; furthermore, these alterations in apoptosis and autophagy were associated with inhibition of the AKT pathway.

Two classical apoptotic pathways were selected to examine the effect of bufadienolides on A-375 cells. First, in the mitochondrial pathway, the Bcl-2 family of proteins exerts a major role in tumorigenesis and tumor maintenance. 
A

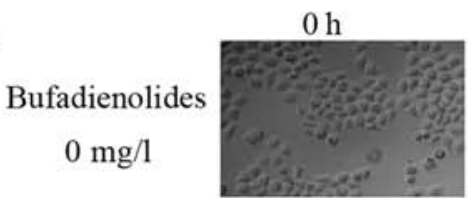

Bufadienolides

$0.001 \mathrm{mg} / 1$

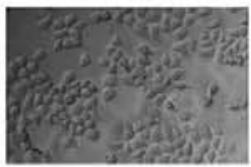

Bufadienolides

$0.01 \mathrm{mg} / 1$

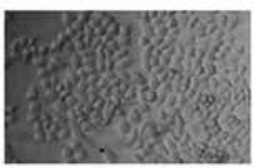

Bufadienolides

$0.1 \mathrm{mg} / 1$

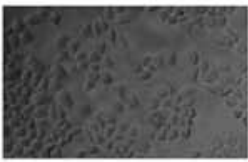

Bufadienolides $1 \mathrm{mg} / 1$
$24 \mathrm{~h}$
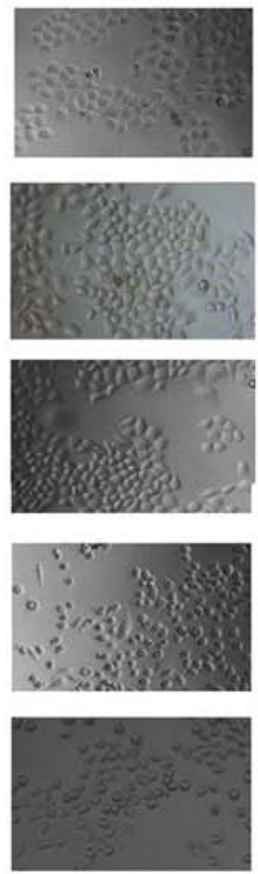

$48 \mathrm{~h}$
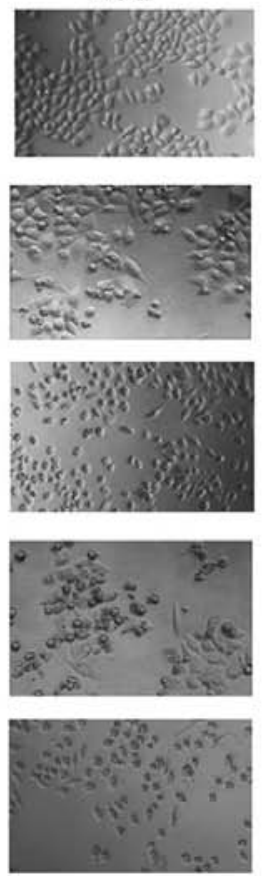

$72 \mathrm{~h}$
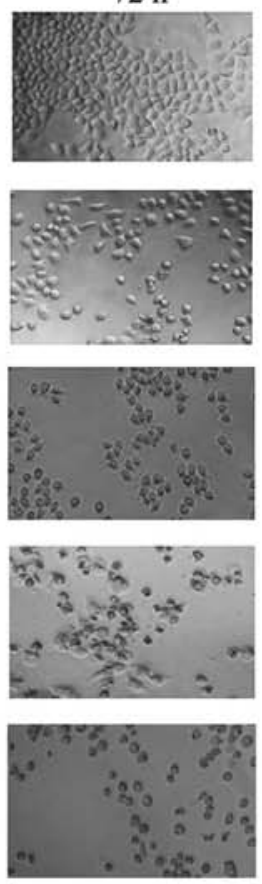
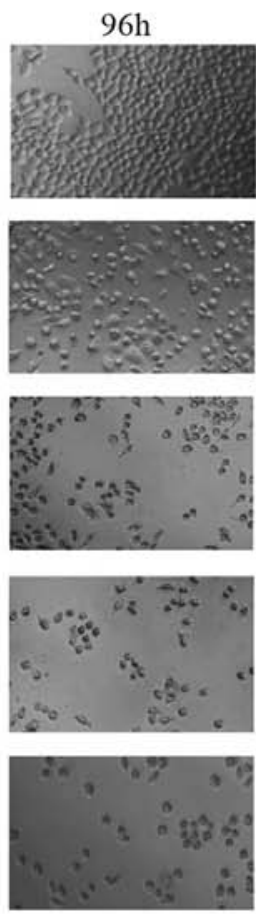
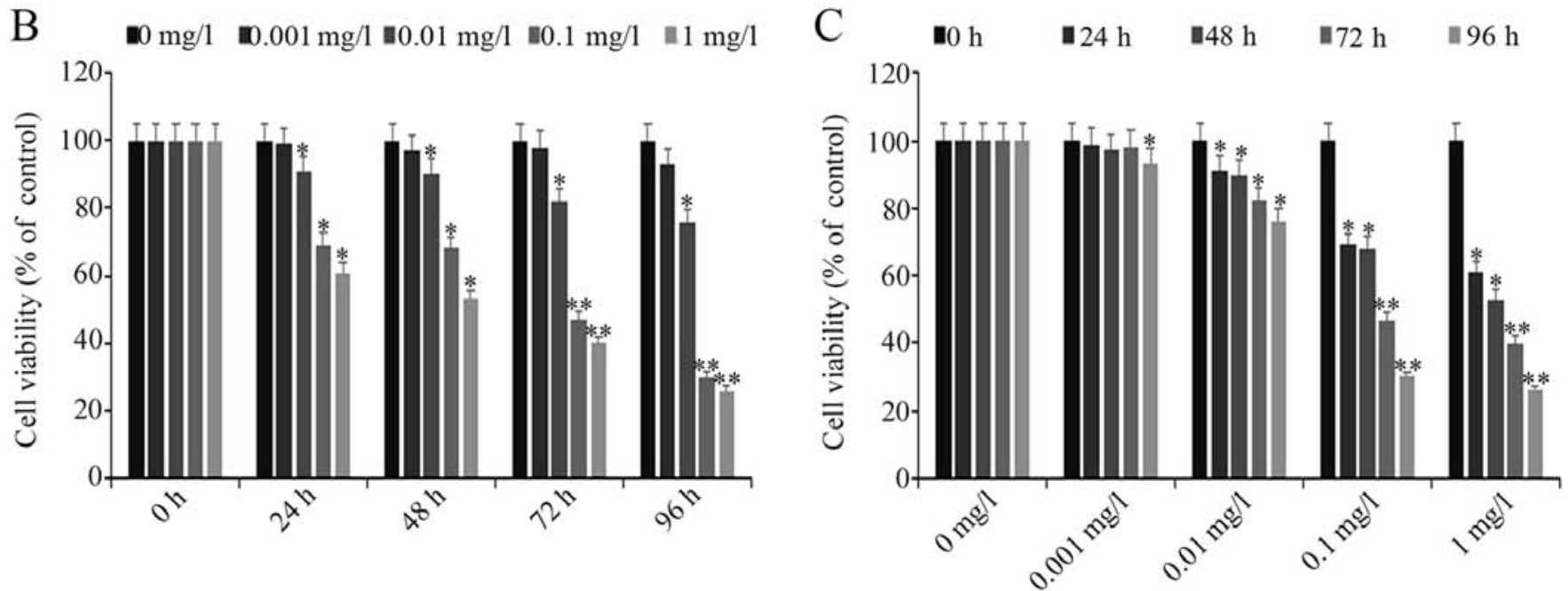

Figure 1. Effects of bufadienolides on A-375 cell viability. (A) Cell morphology changes were observed by phase-contrast microscopy (magnification, x200) after treatment with the various concentrations of bufadienolides for 24, 48, 72 and $96 \mathrm{~h}$. The effects of bufadienolides on A-375 cell viability at (B) different times $(0,24,48,72$ or $96 \mathrm{~h})$ and $(C)$ different concentrations $(0,0.001,0.01,0.1 \mathrm{and} 1 \mathrm{mg} / \mathrm{l})$ are shown. Data are presented as the mean \pm standard deviation of at least three independent experiments. ${ }^{*} \mathrm{P}<0.05$ and ${ }^{* * *} \mathrm{P}<0.01$ vs. respective negative control ( $0 \mathrm{mg} / \mathrm{l}$ bufadienolides).

A previous study revealed that bufadienolides may mediate apoptosis through downregulation of Bcl-2 and/or upregulation of Bax in cancer cells $(24,25)$. In the present study, bufadienolides were identified to induce the apoptosis of A-375 cells, upregulate the expression of Bax and p53, and downregulate Bcl-2 expression. Secondly, the cell death caspases, including initiator caspases and executioner caspases, are known to occupy a central role in the process of apoptosis (26). In the present study, the results also demonstrated that bufadienolides were able to induce apoptosis of A-375 cells via the activation of caspase- 3 .

In addition, autophagy is a cellular catabolic process that helps to maintain cellular homeostasis. The regulation of autophagy exerts an important role in tumor suppression and promotion in numerous types of cancer (27). In cancer therapy, autophagy is activated as an adaptive response to promote cell survival (28). LC3-II is a marker protein for autophagosomes, and increases in the level of this autophagy marker have been demonstrated to inhibit tumor cell growth (29). Furthermore, as an adaptor protein, p62 is able to localize to the site of autophagosome formation and interact with the autophagosome localization protein LC3 to promote tumor cell autophagy $(30,31)$. In the present study, it was observed that bufadienolides upregulated the level of LC3-II, and that p62 was itself degraded by autophagy in a dose-dependent manner.

Accumulating evidence from recent systematic studies has indicated that the AKT pathway exerts an immense influence on the regulation of growth, survival and differentiation of tumor cells (32), and is also associated with their apoptosis 
A
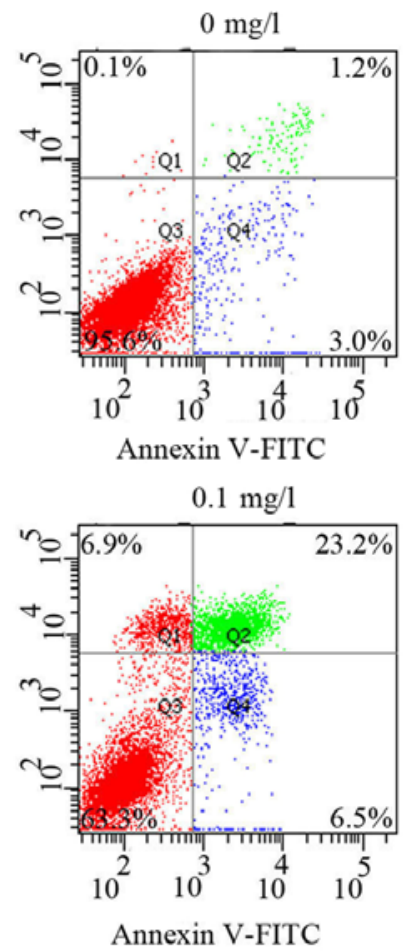
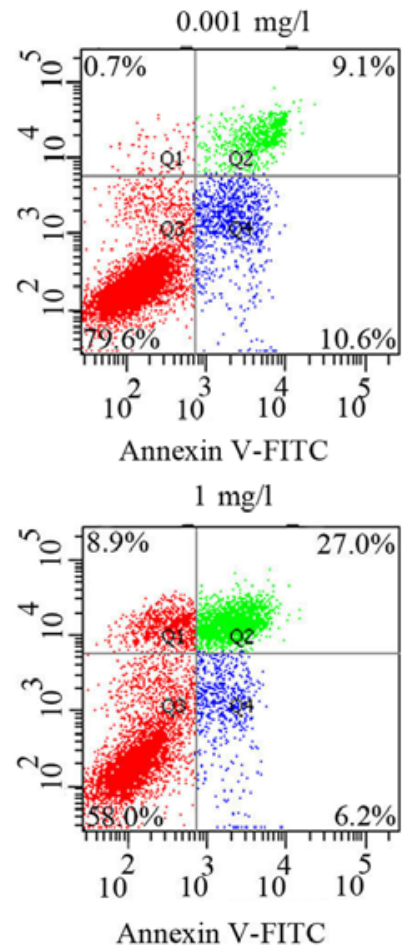
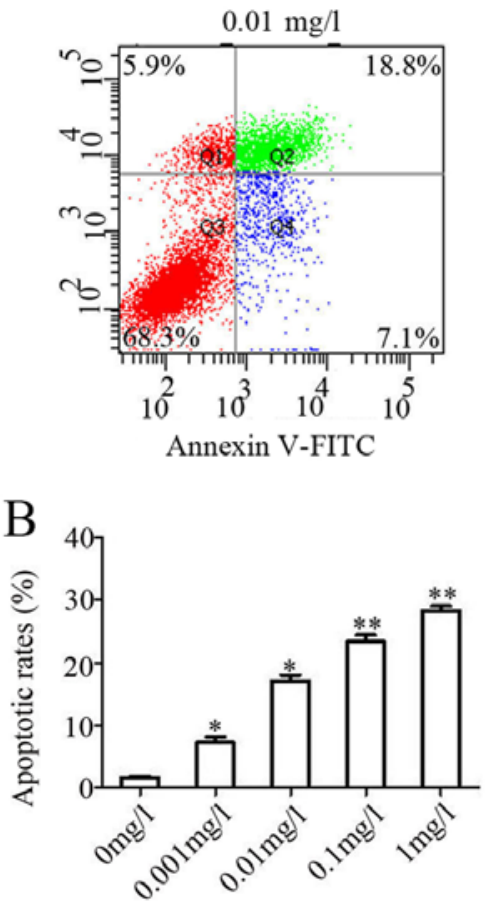
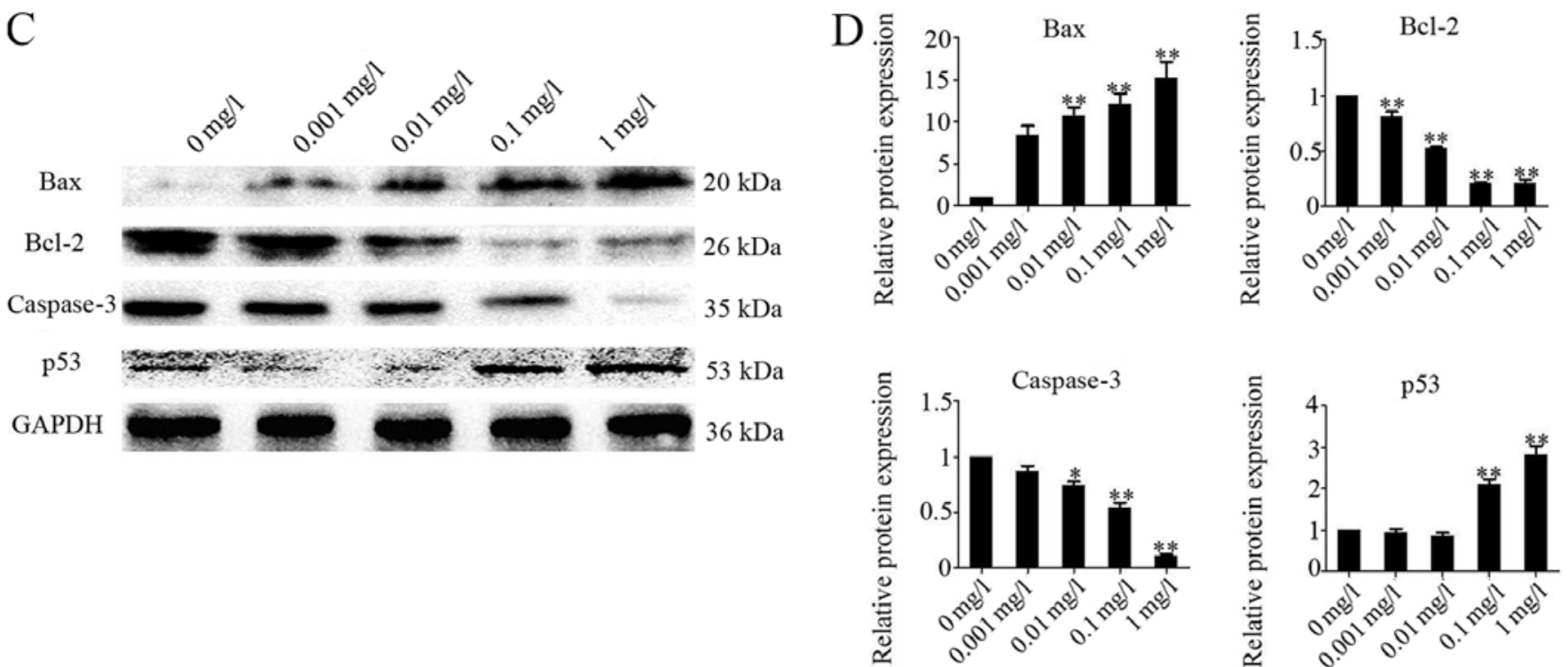

Figure 2. Expression of apoptosis-associated proteins in A-375 cells treated with or without bufadienolides. A-375 cells were treated with bufadienolides $(0,0.001,0.01,0.1$ and $1 \mathrm{mg} / 1)$ for $48 \mathrm{~h}$ in 6 -well plates $\left(2 \times 10^{5} \mathrm{cell} / \mathrm{ml}\right)$. (A) The effects of bufadienolides on the induction of A-375 cell apoptosis were analyzed by flow cytometric analysis. (B) The apoptosis rate was statistically analyzed. (C) Western blotting images for Bax, Bcl-2, caspase-3, p53 and GAPDH in A-375 cells treated with bufadienolides. (D) Quantification of the levels of Bax, Bcl-2, caspase-3 and p53 was performed, and the results were normalized relative to the expression level of GAPDH. Each protein was statistically analyzed, and the results are shown in the right panel. ${ }^{*} \mathrm{P}<0.05$ and ${ }^{* *} \mathrm{P}<0.01$ vs. respective negative control $(0 \mathrm{mg} / \mathrm{l}$ bufadienolides).

and/or autophagy $(33,34)$. In addition, previous studies have shown that inhibition of mTOR is related to the enhancement of apoptosis activation and autophagy induction $(35,36)$. The results of this study showed that the AKT level did not change significantly after treatment of A-375 cells with bufadienolides, but the p-AKT levels decreased, while the mTOR expression level decreased. Inactivated mTOR leads to a decrease in the activity of downstream proteins, p-p70S6K (37) and $\mathrm{p}-\mathrm{GSK}-3 \beta$ (38), and inactivated GSK-3 $\beta$ leads to the inactivation of cyclin D1 (39).
The results indicated that bufadienolides may regulate AKT, p-AKT and proteins located further downstream in the AKT pathway, including mTOR, p-p70S6K, p-GSK-3 $\beta$ and cyclin D1. Based on these results, the present study is the first, to the best of our knowledge, to demonstrate that bufadienolide induces apoptosis and autophagy in A-375 cells, and that this may be due to inhibition of the AKT signaling pathway. Therefore, bufadienolides should be considered as potential drug candidates for the treatment of melanoma by inhibiting the AKT signaling pathway. The limitation of the 
A

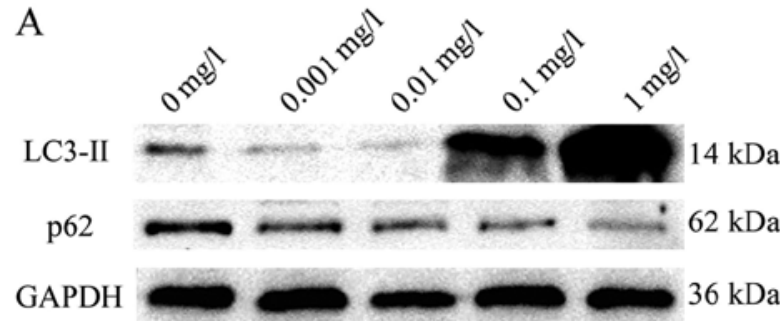

$\mathrm{B}$

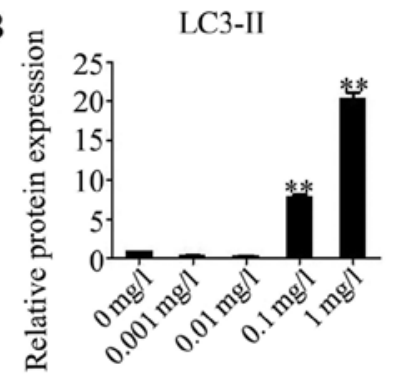

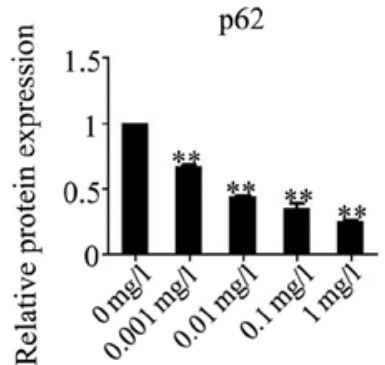

Figure 3. Expression of autophagy-associated proteins in A-375 cells treated with or without bufadienolides. A-375 cells were treated with bufadienolides $(0,0.001,0.01,0.1$ and $1 \mathrm{mg} / 1)$ for $48 \mathrm{~h}$ in 6 -well plates $\left(2 \times 10^{5}\right.$ cell $\left./ \mathrm{ml}\right)$. (A) Western blot images for LC3-II, p62 and GAPDH in A-375 cells treated with bufadienolides. (B) Quantification of the levels of LC3-II and p62 was performed, and the results were normalized relative to the expression level of GAPDH. Each protein was statistically analyzed, and the results are shown to the right of its respective panel. ${ }^{* *} \mathrm{P}<0.01 \mathrm{vs}$. respective negative control ( 0 mg/l bufadienolides). LC3-II, microtubule-associated proteins 1A/1B light chain 3-II.

A

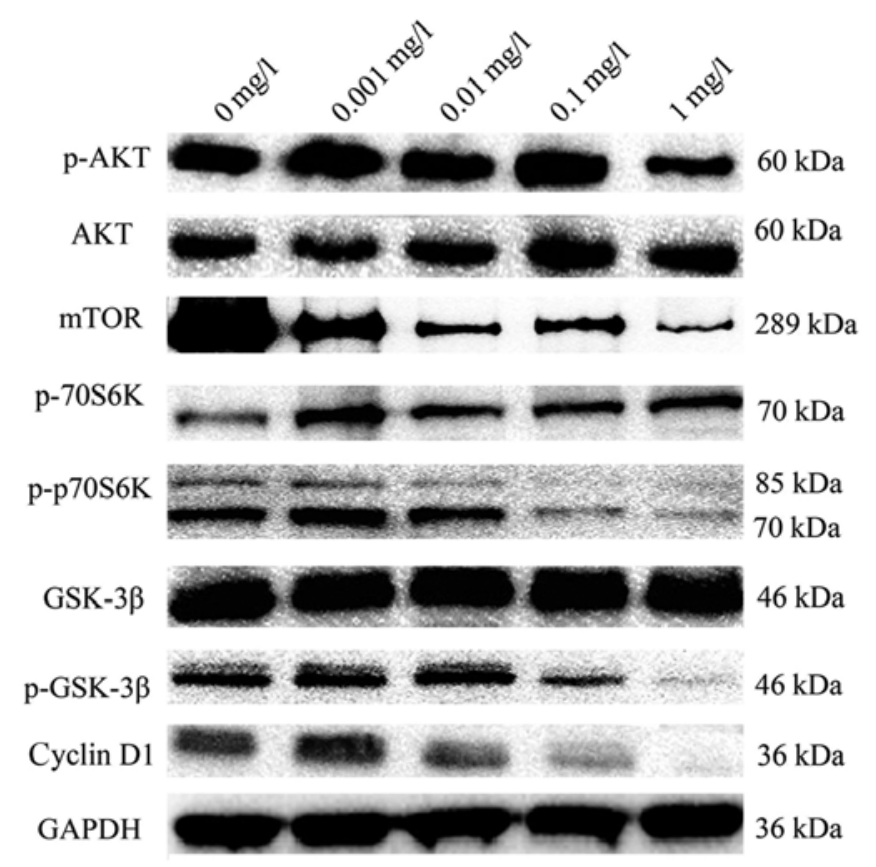

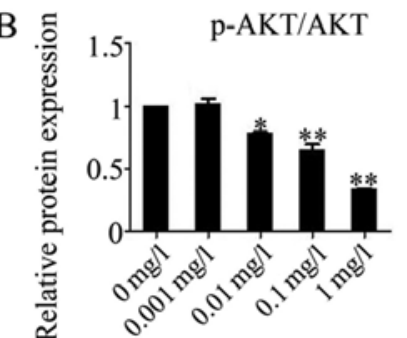

p-p70S6K/p70S6K
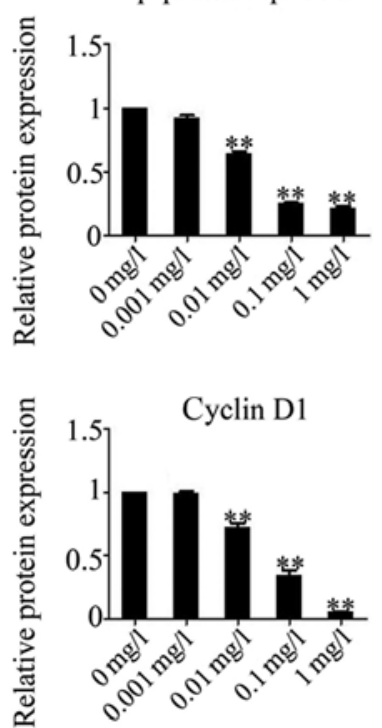

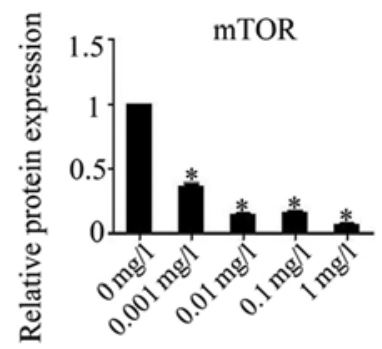

p-GSK-3 $\beta /$ GSK-3 $\beta$

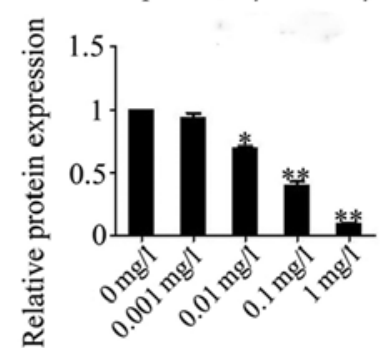

Figure 4. Bufadienolides regulate the AKT signaling pathway in A-375 cells. (A) Western blot images for p-AKT, AKT, mTOR, p70S6K, p-p70S6K, p-GSK-3 $\beta$, p-GSK-3 $\beta$, cyclin D1 and GAPDH in A-375 cells treated with bufadienolides. (B) The relative expression level of each protein following treatment with an increasing concentration of bufadienolides was statistically analyzed. The expression levels for each phosphorylated protein were normalized to the expression levels of the relevant total protein and the loading control. ${ }^{*} \mathrm{P}<0.05$ and ${ }^{* *} \mathrm{P}<0.01$ vs. respective negative control ( 0 mg/l bufadienolides). $\mathrm{p}$, phosphorylated; GSK-3 $\beta$, glycogen synthase kinase- $3 \beta$.

present study is that the effects of bufadienolide treatment were analyzed in only one melanoma cell line, which was not compared with normal epidermal keratinocytes. Further studies in vivo and in vitro in different tumor cell lines and their corresponding normal cell lines are required to demonstrate the anti-tumor mechanism of bufadienolides, which is part of ongoing research. This work will ultimately increase knowledge of the anticancer potential of these naturally occurring compounds.

\section{Acknowledgements}

Not applicable.

\section{Funding}

This study was supported by grants from the Science \& Technology Department of Sichuan Province (grant no. 2019YJ0332) and Chengdu University of TCM (grant no. JSZX2018004). 


\section{Availability of date and materials}

All the data generated or analyzed during the present study are included in this published article.

\section{Authors' contributions}

HL and XC performed the experiments, analyzed the data and wrote the draft version of the manuscript. XL, XC, XY, JX and JC verified the experimental results and revised the manuscript for important intellectual content. LY made substantial contributions to the design of the present study, and critically revised the manuscript.

\section{Ethics approval and consent to participate}

Not applicable.

\section{Patient consent for publication}

Not applicable.

\section{Competing interests}

The authors declare that they have no competing interests.

\section{References}

1. Dountsis A, Zsis C, Karagianni E and Dahabreh J: Primary malignant melanoma of the lung: A case report. World J Surg Oncol 1: 26-29, 2003.

2. Wang Q, Zhang R and Liu D: Long non-coding RNA ZEB1-AS1 indicates poor prognosis and promotes melanoma progression through targeting miR-1224-5p. Exp Ther Med 17: 857-862, 2019.

3. Slominski A, Tobin DJ, Shibahara S and Wortsman J: Melanin pigmentation in mammalian skin and its hormonal regulation. Physiol Rev 84: 1155-1228, 2004.

4. Chang L, Pei J, Li C, Zhang P, Zhou D, Du W, Liu X and Jiang C: Incidence and metastasis of cutaneous malignant melanoma with respect to $\mathrm{ABO}$ blood groups: A case-controlled study in northeast of China. PLoS One 9: e88096, 2014.

5. Sample A and He YY: Mechanisms and prevention of UV-induced melanoma. Photodermatol Photoimmunol Photomed 34: 13-24, 2018.

6. Khan AQ, Travers JB and Kemp MG: Roles of UVA radiation and DNA damage responses in melanoma pathogenesis. Environ Mol Mutagen 59: 438-460, 2018.

7. Miura K and Green AC: Dietary Antioxidants and Melanoma: Evidence from Cohort and Intervention Studies. Nutr Cancer 67 867-876, 2015.

8. Lee SJ, Lee KB, Son YH, Shin J, Lee JH, Kim HJ, Hong AY, Bae HW, Kwon MA, Lee WJ, et al: Transglutaminase 2 mediates UV-induced skin inflammation by enhancing inflammatory cytokine production. Cell Death Dis 8: e3148, 2017.

9. Slominski AT, Brozyna AA, Zmijewski MA, Jóźwicki W, Jetten AM, Mason RS, Tuckey RC and Elmets CA: Vitamin D signaling and melanoma: Role of vitamin D and its receptors in melanoma progression and management. Lab Invest 97: 706-724, 2017.

10. Xiao D, Barry S, Kmetz D, Egger M, Pan J, Rai SN, Qu J, McMasters KM and Hao H: Melanoma cell-derived exosomes promote epithelial-mesenchymal transition in primary melanocytes through paracrine/autocrine signaling in the tumor microenvironment. Cancer Lett 376: 318-327, 2016.

11. Giavina-Bianchi MH, Giavina-Bianchi PF Junior and Festa C Neto: Melanoma: Tumor microenvironment and new treatments. An Bras Dermatol 92: 156-166, 2017.

12. Slominski AT and Carlson JA: Melanoma resistance: A bright future for academicians and a challenge for patient advocates. Mayo Clin Proc 89: 429-433, 2014.
13. Karimkhani C, Green AC, Nijsten T, Weinstock MA, Dellavalle RP, Naghavi M and Fitzmaurice C: The global burden of melanoma: Results from the Global burden of disease study 2015. Br J Dermatol 177: 134-140, 2017.

14. Whiteman DC, Green AC and Olsen CM: The growing burden of invasive melanoma: Projections of incidence rates and numbers of new cases in six susceptible populations through 2031. J Invest Dermatol 136: 1161-1171,2016.

15. Kim JS, Jeong TY, Cho CK, Lee YW and Yoo HS: Antitumor effect of skin of venenum bufonis in a NCIH460 tumor regression model. J Acupunct Meridian Stud 3: 181-187, 2010.

16. Deng LJ, Wang LH, Peng CK, Li YB, Huang MH, Chen MF, Lei XP, Qi M, Cen Y, Ye WC, et al: Fibroblast activation protein $\alpha$ activated tripeptide bufadienolide antitumor prodrug with reduced cardiotoxicity. J Med Chem 60: 5320-5333, 2017.

17. Chang H, Li J, Cao Y, Liu T, Shi S and Chen W: Bufadienolides from venenum bufonis inhibit mTOR-mediated cyclin D1 and retinoblastoma protein leading to arrest of cell cycle in cancer cells. Evid Based Complement Alternat Med 2018: 3247402, 2018.

18. Amaral LS, Martins Ferreira J, Predes D, Abreu JG, Noël F and Quintas LEM: Telocinobufagin and marinobufagin produce different effects in LLC-PK1 cells: A case of functional selectivity of bufadienolides. Int J Mol Sci 19: pii: E2769, 2018.

19. Sheng X, Zhu P, Qin J and Li Q: The biological role of autophagy in regulating and controlling the proliferation of liver cancer cells induced by bufalin. Oncol Rep 39: 2931-2941, 2018.

20. Liu M, Feng LX, Sun P, Liu W, Wu WY, Jiang BH, Yang M, Hu LH, Guo DA and Liu X: A novel bufalin derivative exhibited stronger apoptosis-inducing effect than bufalin in A549 lung cancer cells and lower acute toxicity in mice. PLoS One 11: e0159789, 2016.

21. Zhang H, Zhang X and Zhang J: MiR-129-5p inhibits autophagy and apoptosis of $\mathrm{H} 9 \mathrm{c} 2$ cells induced by hydrogen peroxide via the PI3K/AKT/mTOR signaling pathway by targeting ATG14. Biochem Biophys Res Commun 506: 272-277, 2018.

22. Meng Q, Yau LF, Lu JG, Wu ZZ, Zhang BX, Wang JR and Jiang ZH: Chemical profiling and cytotoxicity assay of bufadienolides in toad venom and toad skin. J Ethnopharmacol 187: 74-82, 2016.

23. Berger MF and Garraway LA: Applications of genomics in melanoma oncogene discovery. Hematol Oncol Clin North Am 23: 397-414, 2009.

24. Cui Y, Zhang Z, Zhang B, Zhao L, Hou C, Zeng Q, Nie J, Yu J, Zhao Y, Gao T, et al: Excessive apoptosis and disordered autophagy flux contribute to the neurotoxicity induced by high iodine in Sprague-Dawley rat. Toxicol Lett 297: 24-33, 2018.

25. Chou HY, Chueh FS, Ma YS, Wu RS, Liao CL, Chu YL, Fan MJ, Huang WW and Chung JG: Bufalin induced apoptosis in SCC-4 human tongue cancer cells by decreasing $\mathrm{Bcl}-2$ and increasing Bax expression via the mitochondria-dependent pathway. Mol Med Rep 16: 7959-7966, 2017.

26. Zaman S, Wang R and Gandhi V: Targeting the apoptosis pathway in hematologic malignancies. Leuk Lymphoma 55: 1980-1992, 2014

27. Yun CW and Lee SH: The roles of autophagy in cancer. Int J Mol Sci 19: pii: E3466, 2018.

28. Radogna F, Dicato M and Diederich M: Cancer-type-specific crosstalk between autophagy, necroptosis and apoptosis as a pharmacological target. Biochem Pharmacol 94: 1-11, 2015.

29. Jena KK, Kolapalli SP, Mehto S, Nath P, Das B, Sahoo PK, Ahad A, Syed GH, Raghav SK, Senapati S, et al: TRIM16 controls assembly and degradation of protein aggregates by modulating the p62-NRF2 axis and autophagy. EMBO J 37: pii: e98358, 2018

30. Niklaus M, Adams O, Berezowska S, Zlobec I, Graber F, Slotta-Huspenina J, Nitsche U, Rosenberg R, Tschan MP and Langer R: Expression analysis of LC3B and p62 indicates intact activated autophagy is associated with an unfavorable prognosis in colon cancer. Oncotarget 8: 54604-54615, 2017.

31. Sophia J, Kowshik J, Dwivedi A, Bhutia SK, Manavathi B, Mishra R and Nagini S: Nimbolide, a neem limonoid inhibits cytoprotective autophagy to activate apoptosis via modulation of the PI3K/Akt/GSK-3 $\beta$ signalling pathway in oral cancer. Cell Death Dis 9: 1087, 2018.

32. Sun YZ, Cai N and Liu NN: Celecoxib down-regulates the hypoxia-induced expression of HIF-1 $\alpha$ and VEGF through the PI3K/AKT pathway in retinal pigment epithelial cells. Cell Physiol Biochem 44: 1640-1650, 2017.

33. Feng H, Cheng X, Kuang J, Chen L, Yuen S, Shi M, Liang J, Shen B, Jin Z, Yan J and Qiu W: Apatinib-induced protective autophagy and apoptosis through the AKT-mTOR pathway in anaplastic thyroid cancer. Cell Death Dis 9: 1030, 2018. 
34. Fan YP, Liu P, Xue WK, Zhao WJ and Pan HC: Trimebutine promotes Glioma cell apoptosis as a potential anti-tumor agent. Front Pharmacol Front Pharmacol 9: 664, 2018.

35. Shen S, Zhang Y, Wang Z, Liu R and Gong X: Bufalin induces the interplay between apoptosis and autophagy in glioma cells through endoplasmic reticulum stress. Int J Biol Sci 10: 212-224, 2014.

36. Zhang DM, Liu JS, Deng LJ, Chen MF, Yiu A, Cao HH, Tian HY, Fung KP, Kurihara H, Pan JX and Ye WC: Arenobufagin, a natural bufadienolide from toad venom, induces apoptosis and autophagy in human hepatocellular carcinoma cells through inhibition of PI3K/Akt/mTOR pathway. Carcinogenesis 34: $1331-1342,2013$
37. Kim SJ, Kim JH, Jung HS, Lee TJ, Lee KM and Chang IH: Phosphorylated p70S6K in noninvasive low-grade urothelial carcinoma of the bladder: Correlation with tumor recurrence. Asian J Androl 16: 611-617, 2014.

38. Kaidanovich-Beilin O and Woodgett JR: GSK-3: Functional Insights from Cell Biology and Animal Models. Front Mol Neurosci 4: 40, 2011.

39. Wang Q, Zheng M, Yin Y and Zhang W: Ghrelin stimulates hepatocyte proliferation via regulating cell cycle through GSK3 $/$ /B-catenin signaling pathway. Cell Physiol Biochem 50: 1698-1710, 2018. 